\title{
Research on Intelligent Following Motion Control System of Rehabilitation Robot
}

\section{Mingda Miao (D845771325@qq.com )}

Beijing Institute of Technology https://orcid.org/0000-0002-7518-9165

\section{Xueshan Gao}

Beijing Institute of Technology School of Mechatronical Engineering

Jun Zhao

China Rehabilitation Research Center

\section{Peng Zhao}

Guangxi University of Science and Technology

\section{Research}

Keywords: Lower limb rehabilitation robot, Motion data analysis, Dynamic model, Radial basis function neural network, Adaptive sliding mode control

Posted Date: December 1st, 2021

DOI: https://doi.org/10.21203/rs.3.rs-1074506/v1

License: (c) (1) This work is licensed under a Creative Commons Attribution 4.0 International License. Read Full License 


\title{
Research on Intelligent following motion control system of rehabilitation robot
}

\author{
Ming da Miao ${ }^{*}$, Xue shan Gao ${ }^{1,3}$, Jun Zhao ${ }^{2}$ and Peng Zhao ${ }^{3}$
}

\author{
${ }^{*}$ Correspondence: \\ 845771325@qq.com \\ ${ }^{1}$ School of Mechatronical \\ Engineering, Beijing Institute of \\ Technology, Beijing, China \\ Full list of author information is \\ available at the end of the article
}

\begin{abstract}
Background: In response to the current problem of low intelligence of mobile lower limb motor rehabilitation aids, this article proposes an intelligent control scheme based on human movement behavior in order to control the rehabilitation robot to follow the patient's movement.

Methods: Firstly, a multi-sensor data acquisition system is designed according to the motion characteristics of human body. By analyzing and processing the motion data, the change law of human center of gravity and behavior intention are obtained, and the behavior intention of human is used as the control command of the robot following motion. In order to achieve the goal of the rehabilitation robot following human motion, an adaptive radial basis function neural network sliding mode controller is designed based on the robot dynamic model. The controller can reduce the impact of fluctuations in the human center of gravity on changes in the parameters of the robot control system, and enhance the adaptability of the system to other disturbance factors, and improve the accuracy of following human motion. Finally, the motion following experiment of the rehabilitation robot is carried out.

Results: The experimental results show that the robot can recognize the motion intention of human body, and achieve the training goal of following different subjects to complete straight lines and curves.
\end{abstract}

Results: According to the experimental results, the accuracy of the multi-sensor data acquisition system and control algorithm design is verified, which demonstrates the feasibility of the proposed intelligent control scheme.

Keywords: Lower limb rehabilitation robot; Motion data analysis; Dynamic model; Radial basis function neural network; Adaptive sliding mode control

\section{Background}

With the rapid development of science and technology, traditional medical equipment is constantly developing in the direction of intelligence. Medical robots are a combination of multi-disciplinary development achievements. They are widely used in medical diagnosis and treatment, clinical surgery, rehabilitation medicine and other related medical fields. This section is divided into subheadings. It provides a detailed description of the experimental results, their interpretation, as well as the experimental conclusions that can be drawn [1]. Due to the aggravation of social aging, the number of patients with motor dysfunction caused by stroke or other diseases, significantly increased $[2,3]$. As a kind of robots used in the field of rehabilitation medicine, the rehabilitation robot can help patients with exercise or cognitive function training. In addition, it can solve, to a certain extent, the prob- 
lems of fatigue and differences in multiple training, in artificial rehabilitation treatment [4]. However, most of the rehabilitation robots only provide exercise function training or cognitive function training for patients. The interactive control between the rehabilitation robot and patients is essential, due to the fact that patients have autonomous consciousness [5]. Human computer interaction control can provide patients with good personal adaptability and active compliance in rehabilitation treatment. It also improves the training comfort, safety and rehabilitation effect in gait rehabilitation [6]. Moreover, the human-computer interaction system can supplement the gait rehabilitation plan of patients, reduce the workload of doctors, give patients motivation and encouragement in the treatment process, and improve the patient participation and performance in the treatment process $[7,8,9]$. The rehabilitation robot training method is based on the combination of motion and cognition. The control strategy will become a new research hotspot in the field of rehabilitation robots $[10,11]$.

In order to improve the control effect of the robot in human-computer interaction, the authors in $[12,13,14]$ studied the control strategy of the lower limb exoskeleton robot. The latter improves the comfort of rehabilitation treatment, enhances the anti-interference of the system and the speed of tracking the desired trajectory, and provides assistance for the recovery of lower limb motor function of stroke patients. The authors in [15] designed a lower limb rehabilitation robot for gait and balance training of stroke patients. This robot can provide weight support for patients, maintain patient balance and track patient movement. Several control methods of lower limb rehabilitation robots cannot adapt to different gait cycles and rehabilitation treatment modes. The authors in [16] proposed a torque auxiliary control method based on sliding mode adaptive control. The author uses a radial basis function neural network algorithm to learn the auxiliary torque required for normal gait trajectory. Consequently, the rehabilitation robot can adaptively provide auxiliary torque according to the needs of subjects. Several researchers proposed various control schemes for mobile robot trajectory tracking. For instance, in [17], an adaptive sliding mode control scheme is proposed to allow the mobile robot to achieve ideal speed control under uncertain disturbance system. However, it does not consider the influence of position disturbance, and the robustness of the control system is very poor. In [18], a sliding mode controller based on robust neural network, is proposed. The controller uses a neural network to directly identify the dynamic characteristics of the system, overcomes the shortcomings of integral sliding mode control and improves the system robustness. An adaptive neural sliding mode dynamic trajectory tracking control scheme is proposed in $[19,20]$. It can smooth the control input and eliminate the tracking error in the case of persistent disturbance and uncertainty. This system has the advantages of fast convergence and strong robustness. By analyzing the characteristics of human motion behavior, combined with the human-computer interaction and mobile robot technologies, this paper studies the motion control strategy of the human lower limb rehabilitation robot. It also designs a wearable data acquisition device based on a multi-sensor system, processes the motion information and extracts the motion characteristics. Afterwards, based on the dynamic model, an adaptive radial basis function neural network (ARBFNN) sliding mode controller is designed. Using the characteristics 
of on-line adaptive learning of ARBFNN, the switching gain of the dynamic controller is modified in real time, in order to enhance the adaptability to the changes of the system parameters and other disturbance factors. Consequently, the robot can follow the subject along the set trajectory after identifying the human motion intention.

The remainder of this paper is organized as follows. In section "Methos", some problems faced by patients in the current rehabilitation field are described. The overall control scheme of intelligent follow-up system is then proposed. In addition, the motion characteristics of human walking are analyzed, a multi-sensor information system based on the analysis results is designed, and the processing method and application of motion data is developed. Moreover, based on the dynamic equation of rehabilitation robot, a ARBFNN sliding mode controller is designed, and its stability is analyzed. In section "Results", the experimental scheme of following motion control is designed, and the experimental data are briefly analyzed. In section "Discussion", the experimental results are detailed and analyzed, and the shortcomings of the proposed method are reviewed. Finally, conclusions are drawn in section "Conclusions".

\section{Methods}

System scheme design

Problem description

Stroke patients generally choose to be treated in hospitals or rehabilitation institutions. Most of the rehabilitation equipment in institutions are fixed, while few intelligent mobile rehabilitation equipment exists. In addition, the number of doctors is also insufficient, resulting in many patients waiting in line. Moreover, due to the decline of quality of life, many patients become grumpy and difficult to adhere to the rehabilitation process. With the assistance of an intelligent mobile rehabilitation robot, a doctor can care for and guide multiple patients for rehabilitation simultaneously. This not only improves the rehabilitation efficiency, but also reduces the doctor's workload and reduces the patient's waiting time. In addition, the real walking rehabilitation training experience can enhance the patient's subjective rehabilitation will, and allow the patient to actively participate in the rehabilitation treatment.

\section{Overall system design}

The system of the rehabilitation robot platform is shown in Figure 1(a). The robot system consists of four parts: multi-sensor, control, weight support and driving systems [21]. Firstly, the user stands in the middle of the rehabilitation robot and wears auxiliary clothes to connect with the rehabilitation robot. During use, the weight support system provides auxiliary support for the user, in order to reduce the burden of lower limb movement when the user walks. The multi-sensor system senses the motion data of the human body and transmits the data to the control system. The latter sends the control command to the driving system, to make the robot follow the human body. The overall control system structure of the rehabilitation robot is presented in Figure 1(b). 


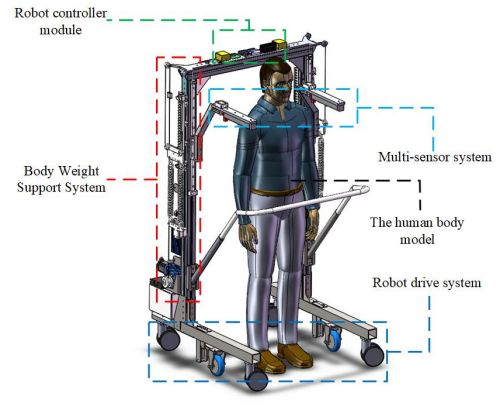

(a)

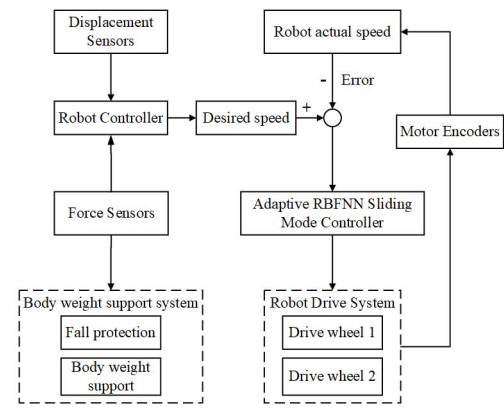

(b)

Figure 1 (a) Structure design of the rehabilitation robot (b) Structure of the control system

Design and data analysis of the multi-sensor information system

People's body posture changes periodically and regularly when walking. Everyone's posture change law is different and has its own unique form, due to the individual differences in height, weight, step frequency and step length. The more obvious posture changes are the up and down fluctuations of the human center of gravity and the left and right swing of the shoulder, as shown in Figures 2 and 3. Although the body posture of stroke patients is different from that of healthy people, periodic and regular characteristics still exist when walking. Therefore, it is necessary to process the posture data of human motion and extract the regular characteristics. This section introduces the design and data feature extraction method of the multisensor motion data information acquisition system.

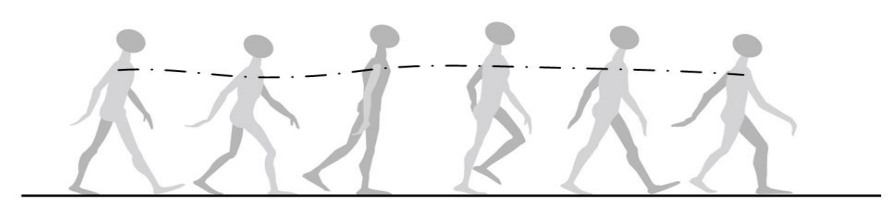

Figure 2 Posture change of human motion center of gravity

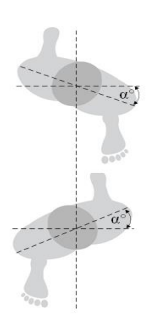

(a)

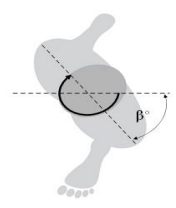

(b)

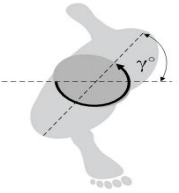

(c)

Figure 3 Posture changes of human shoulder (a) straight forward (b) turn right (c) turn left 
Design of the motion data acquisition system

The multi-sensor system collects the center of gravity fluctuation and shoulder displacement data with obvious changes during human walking. It is necessary to select the sensor used for sensing the human behavior, and determine the type, quantity and installation position of the sensor according to the characteristics of the human walking posture, in order to obtain accurate human motion data. The scheme of the multi-sensor motion data acquisition system is shown in Figures 4 and 5. The system includes two displacement sensors and two tension sensors.

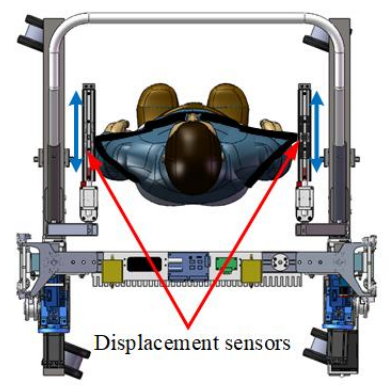

(a)

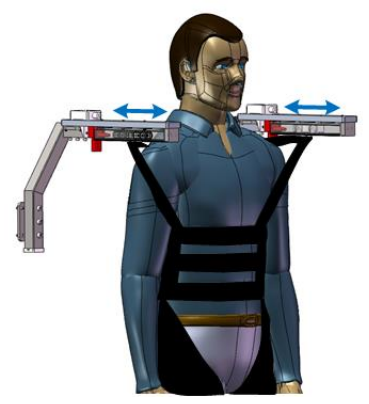

(b)

Figure 4 Schematic diagram of a dual displacement sensor (a) top view (b) axonometric view

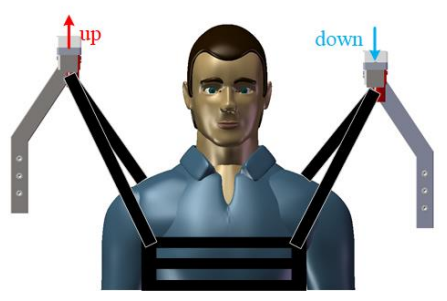

(a)

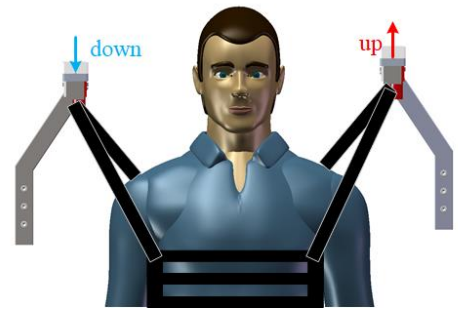

(b)

Figure 5 Schematic diagram of a double tension sensor (a) walking on the right side of limb (b) walking on the left side of limb

The system uses the MPS-XXXS pull rope displacement sensor, with a displacement accuracy of $0.1 \mathrm{~mm}$ and a displacement stroke range of 0-200 $\mathrm{mm}$. The Dyly106 tension sensor is used with a range of $0-50 \mathrm{~kg}$ and a tension accuracy of $0.03 \mathrm{~kg}$. Both sensors have the advantages of high precision, fast response and long service life, and meet the actual working conditions. The data collected by the displacement and tension sensors are digital. The motion data is sent to MCU through RS-485. The sampling frequency of the sensor is $200 \mathrm{~Hz}$. The feedback data of the displacement and tension sensors is the basis for the subsequent controller design and robot motion control [21]. 
Motion data analysis and processing

Firstly, the displacement of the human shoulder is taken as the external control input of the rehabilitation robot overall system. The linear relationship between the displacements $x_{1}$ and $x_{2}$ of the displacement sensor and the driving wheel speeds $v_{1}$ and $v_{2}$ of the rehabilitation robot, is established. The speed $V_{c}$ obtained from the linear relationship is approximately considered as the motion speed of the human body. It is taken as the expected input of the internal controllers in the robot. If the robot can accurately follow the desired motion speed, it can achieve the control goal of following human motion.

$$
\left\{\begin{array}{l}
v_{1}=\lambda \times \mathrm{x}_{1} \\
v_{2}=\lambda \times \mathrm{x}_{2}
\end{array}\right.
$$

where $\lambda$ is the constant coefficient between the displacement and driving wheel speed.

Since the rehabilitation robot needs to provide auxiliary force for stroke patients, the dynamic parameter $m$ of the robot will change according to the auxiliary force. Because parameter $m$ directly affects the control effect of the rehabilitation robot controller, the law and characteristics of the center of gravity change in the process of human walking are studied. Data support is also provided for the subsequent controller design. The initial values of the auxiliary force are set as $F_{1}$ and $F_{2}$. With the change of the center of gravity of the human body during walking, the actual auxiliary force values fed back by the tension sensor are $F_{1 \mathrm{r}}$ and $F_{2 \mathrm{r}}$. The change values of the auxiliary force on both sides of the human body are then:

$$
\left\{\begin{array}{c}
\Delta F_{1}=F_{1 r}-F_{1} \\
\Delta F_{2}=F_{2 r}-F_{2}
\end{array}\right.
$$

The variation values of the auxiliary forces on both sides of the human body are treated and considered as the variation characteristics of the center of gravity of the human body. The variation values of this point are given by:

$$
\Delta m=\frac{\Delta F_{1}+\Delta F_{2}}{2}
$$

In equations (2) and (3), $\Delta F_{1}$ and $\Delta F_{2}$ are the auxiliary forces changes on the left and right sides of the human body, respectively.

Correctness verification of data feature extraction

The human gravity center change data collected by the Minisun-IDEEA gait analyzer is used as reference standard, in order to verify the correctness of the human gravity center change data processing method [22, 23, 24, 25]. Firstly, a healthy adult volunteer is selected as the object of human center of gravity data acquisition. The motion data of volunteers are then collected by the IDEEA gait analyzer and rehabilitation robot data acquisition system. The change curve of the human center of gravity, obtained by gait analyzer, is shown in Figure 6 . The change curve of auxiliary power on the left and right sides of the human body, obtained by the robot data acquisition system, is presented in Figure 7. 


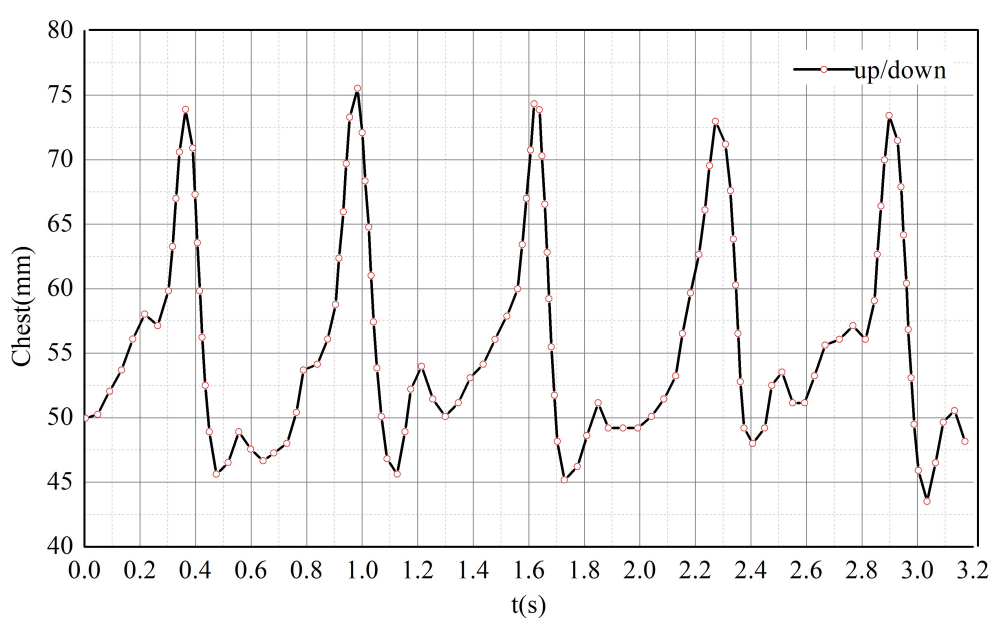

Figure 6 Fluctuation curve of human gravity center

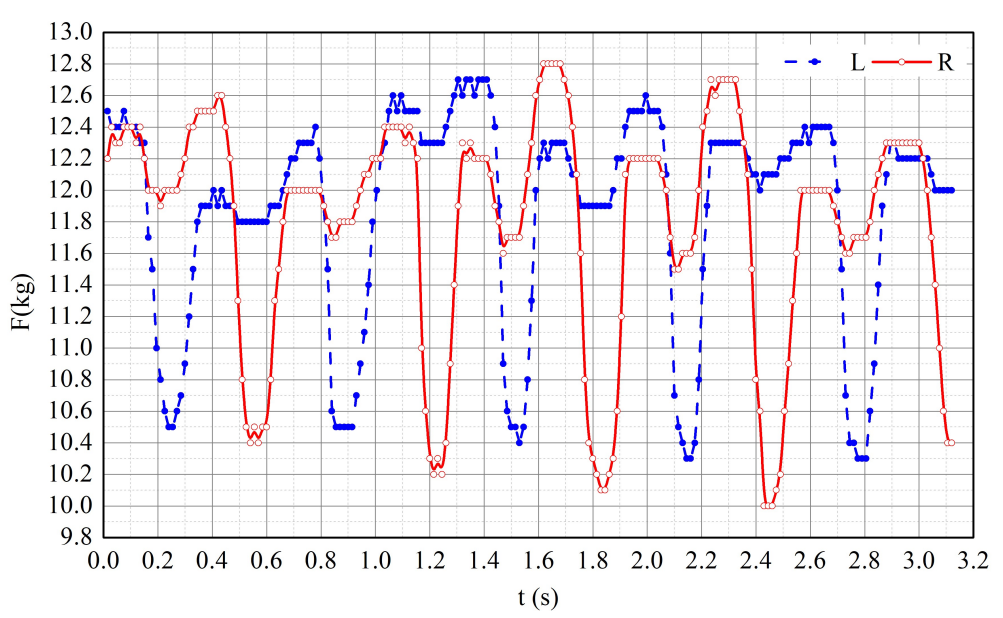

Figure 7 Variation curve of left and right auxiliary forces of human body

It can be seen by comparing the human motion data in Figures 6 and 7 that, when the center of gravity of the human body moves up, the auxiliary force of the robot decreases. On the contrary, when the center of gravity moves down, the auxiliary force increases. Therefore, the auxiliary force curves on the left and right sides of the human body have the opposite characteristics with the center of gravity curve. Since the units of the gravity center displacement value and auxiliary force value are different, the human motion data collected by the robot, are uniformly processed. It can be seen from Figure 8 that the displacement behavior of the left and right sides of the human body, and the reference displacement of the gravity center, are approximately the same. The human weight center displacement obtained by the gait analyzer is almost $30 \mathrm{~mm}$. The human body displacement collected by the robot is almost $25 \mathrm{~mm}$ on the left side, while that on the right side is almost 27.5 $m m$ with a small error, which proves the reliability of the data acquisition system design and the correctness of feature extraction. 


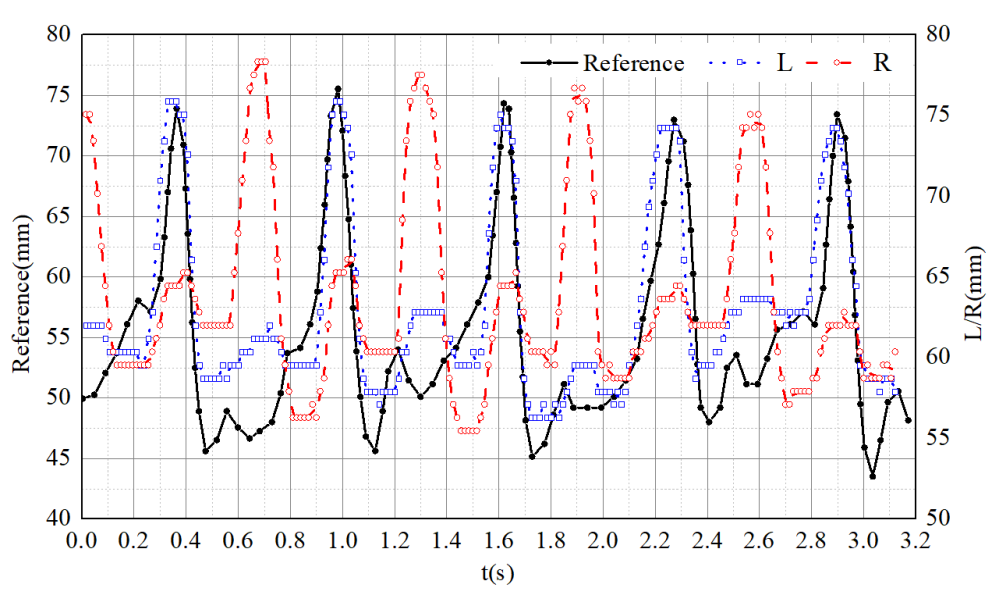

Figure 8 Comparison curve of human gravity center displacement

Finally, the Fourier function first-order polynomial is used to approximate the function expression of the auxiliary force change curve. The auxiliary force change fitting curve is shown in Figure 9. The obtained relationship is expressed as:

$$
f(x)=a_{0}+a_{1} \cos (x \times w)+b_{1} \sin (x \times w)
$$

where $a_{0}, a_{1}, b_{1}$ and $w$ are the fitting function coefficients.

In the subsequent design of rehabilitation robot controller, the result of this function is considered as the disturbance factor in the robot system.

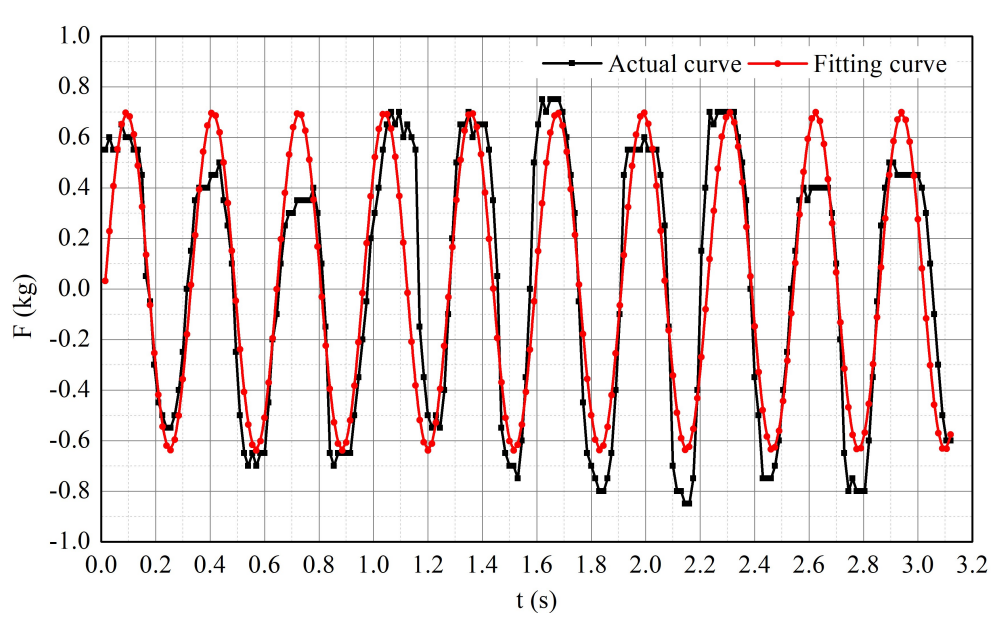

Figure 9 Comparison curve of human gravity center displacement

\section{Dynamics modeling of the rehabilitation robot}

The research object of this paper is a mobile rehabilitation robot with six wheels structure. As shown in Figure 10, this robot is composed of two central driving wheels and four front and rear universal wheels installed on the same axis [21]. 
Vector $(x, y, \theta)^{T}$ represents the posture of the rehabilitation robot in the coordinate system $O X Y$, where $(x, y)$ represents the coordinates of the rehabilitation robot reference point $p, \theta$ denotes the steering angle of the rehabilitation robot. The spacing of the driving wheels is $2 b$, while the radius of the driving wheels is $r$.

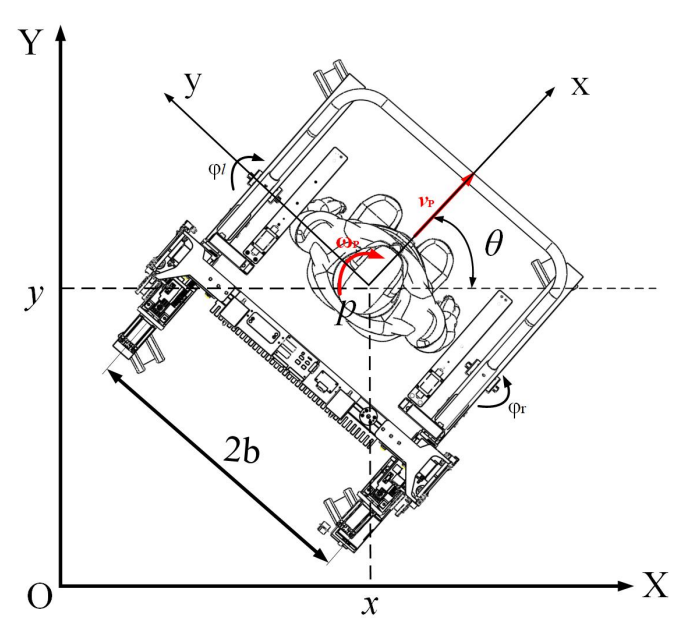

Figure 10 Schematic diagram of the rehabilitation robot

The rotational angular speeds of the left and right driving wheels of the rehabilitation robot are $\varphi_{l}$ and $\varphi_{r}$. Let $V(t)=[v, w]^{T}$, where $v$ and $\mathrm{w}$ are respectively used to represent the linear and angular speed of the rehabilitation robot point $p$. The motion speed of the rehabilitation robot is given by:

$$
V(t)=\left[\begin{array}{c}
v \\
w
\end{array}\right]=\left[\begin{array}{cc}
\frac{r}{2} & \frac{r}{2} \\
\frac{r}{2 b} & -\frac{r}{2 b}
\end{array}\right]\left[\begin{array}{l}
\varphi_{l} \\
\varphi_{r}
\end{array}\right]
$$

The dynamic model of the rehabilitation robot is the most essential model, and it can best reflect its motion characteristics. The dynamic equation of the rehabilitation robot is given by:

$$
\bar{M}(q) \dot{V}+\bar{C}(q, \dot{q}) V+\bar{\tau}_{d}=\bar{B}(q) \tau
$$

According to Equation (6):

$$
\dot{V}=\bar{M}^{-1}(q)\left[\bar{B}(q) \tau-\bar{\tau}_{d}\right]=B_{0} \tau-\bar{M}^{-1}(q) \bar{\tau}_{d}
$$

where $\bar{M}(q)$ is the inertia matrix, $\bar{C}(q, \dot{q})$ is the Coriolis force and centripetal force matrix, $V(t)=\left[v_{p}, w_{p}\right]^{T}$ is a generalized velocity vector, $\bar{\tau}_{d}$ is the unknown disturbance of bounded unmodeled dynamics, $\bar{B}(q)$ is a nonsingular matrix related only to the wheel spacing $b$ and wheel radius $r$, and $\tau$ is the input of the driving wheel torque control of the rehabilitation robot.

Each matrix is taken as: 


$$
\begin{gathered}
\bar{M}(q)=\left[\begin{array}{cc}
m & 0 \\
0 & I
\end{array}\right] ; \quad \bar{C}(q, \dot{q})=\left[\begin{array}{cc}
0 & 0 \\
0 & 0
\end{array}\right] ; \quad \bar{B}(q)=\frac{1}{r}\left[\begin{array}{cc}
1 & 1 \\
b & -b
\end{array}\right] ; \quad \tau= \\
{\left[\begin{array}{c}
\tau_{1} \\
\tau_{2}
\end{array}\right] ; \bar{M}^{-1}(q)=\frac{1}{m I}\left[\begin{array}{cc}
I & 0 \\
0 & m
\end{array}\right] ; \quad B_{0}=\bar{M}^{-1}(q) \bar{B}(q)=\frac{1}{m r I}\left[\begin{array}{cc}
I & I \\
m b & -m b
\end{array}\right]}
\end{gathered}
$$

where $m$ is the sum of the rehabilitation robot $m_{1}$ and the patient's auxiliary force $m_{2}, I$ is the central moment of inertia of the rehabilitation robot (including the patient), $\tau_{1}$ and $\tau_{2}$ are the torque of the left and right drive wheels, respectively.

By ignoring the uncertain interference $\left(\bar{\tau}_{d}=0\right)$, Equation (7) is simplified as:

$$
\dot{V}=B_{0} \tau
$$

\section{Design of ARBFNN sliding mode controller}

\section{Controller design}

The rehabilitation robot should provide auxiliary force for patients. Part of the weight of the patient's body is applied to the robot as a load. Different patients need different auxiliary forces and different loads on the robot. Therefore, the dynamic parameter $m$ of the robot changes. In this paper, combined with the velocity input of human body and the torque input of dynamics, the sliding mode concept is used to design the controller for the system dynamics in part (6). the influence of the change of center of gravity on parameter $\mathrm{m}$ is considered as a disturbance. In addition, the control algorithm is used to compensate its influence on the control effect of the robot, so that the speed of the rehabilitation robot converges to the

expected speed given by human motion. The desired input of the robot is calculated according to the motion speed of the human body:

$$
V_{c}=\left[\begin{array}{c}
v_{c} \\
w_{c}
\end{array}\right]=\left[\begin{array}{c}
\left(v_{1}+v_{2}\right) / 2 \\
\left(v_{1}-v_{2}\right) / 2 b
\end{array}\right]
$$

The speed following error is defined as:

$$
e_{c}=V_{c}-V=\left[\begin{array}{l}
e_{1} \\
e_{2}
\end{array}\right]=\left[\begin{array}{c}
v_{c}-v \\
w_{c}-w
\end{array}\right]
$$

The sliding surface,selected according to equation(8), is given by:

$$
S=\left[\begin{array}{l}
s_{1} \\
s_{2}
\end{array}\right]=e_{c}+\eta \int_{0}^{t} e_{c} d \tau
$$

where $\eta$ is the sliding mode area fraction constant and $\eta>0$.

The derivative of equation (11) can be computed as:

$$
\dot{S}=\dot{e}_{c}+\eta e_{c}=\dot{V}_{c}-B_{0} \tau+\eta e_{c}
$$

Let $T=B_{0} \tau$ as a new control variable, in order to make the state trajectory stay on the sliding mode surface:

$$
\left.\dot{S}\right|_{T=T_{e q}}=0
$$


When the system parameters are determined, the equivalent control rate $T_{e q}$ is expressed as:

$$
T_{e q}=B_{0}^{-1}\left(\dot{V}_{c}+\eta e_{c}\right)
$$

Equivalent control law $\tau_{\text {eq }}$ can make the system stable on the sliding mode surface, because the system can be adjusted by $\eta$ values to change its control characteristics. However, due to the uncertainty and external disturbance of the system dynamic parameter $m$, a discontinuous control law is introduced to meet the sliding condition $\tau_{\mathrm{sw}}$, in order to enhance the system robustness. Finally, the integral sliding mode motion following the control law, is determined by the equivalent control law $\tau_{\text {eq }}$ and switching control law $\tau_{\text {sw }}$ composition:

$$
\tau=\tau_{e q}+\tau_{s w}=B_{0}^{-1}\left(\dot{V}_{c}+\eta e_{c}+\xi \operatorname{sgn}(S)\right)
$$

where $B_{0}^{-1}=\frac{r}{2 b}\left[\begin{array}{cc}m b & I \\ m b & -I\end{array}\right], \xi=\left[\begin{array}{cc}\xi_{1} & 0 \\ 0 & \xi_{2}\end{array}\right], \operatorname{sgn}(S)=\left[\operatorname{sgn}\left(s_{1}\right), \operatorname{sgn}\left(s_{2}\right)\right]^{T}$, and $\xi$ is a normal number.

By considering the uncertainty and other external disturbances caused by the change of human gravity center on the dynamic parameter $m$, equation (8) can be written as:

$$
\dot{V}=\left(B_{0}+\Delta B_{0}\right) \tau+\delta(t)
$$

where $B_{0}$ is the nominal part of the system matrix, determined by parameters $r, b$, $m$ and the moment of inertia $I$ of the robot, $\Delta B_{0}$ is the internal disturbed part of the system matrix, determined by the dynamic parameters $\Delta m$ and $\Delta I$ caused by the change of the center of gravity of the human body, $\delta(t)$ is an external disturbance vector, including plane friction and disturbance torque, whose specific value is difficult to be determined in practical applications.

In order to meet the optimal speed tracking and achieve the performance of the system, the radial basis function neural network (RBFNN) is introduced to adjust the sliding mode switching gain $\xi$. The sliding surface is defined as the input of the RBFNN. The output for an input $x_{\mathrm{i}}=\left[\mathrm{s}_{1}, \mathrm{~s}_{2}\right], i=1,2$ is given by:

$$
\xi_{i}=W_{i}^{T} H_{i}=w_{i 1} h_{i 1}+w_{i 2} h_{i 2}+\cdots+w_{i n} h_{i n}
$$

where $W_{i}=\left[w_{i 1} w_{i 2} \cdots w_{i n}\right]^{T}$ is the weight vector of the neural network, $H_{i}=$ $\left[h_{i 1} h_{i 2} \cdots h_{i n}\right]^{T}$ is the radial basis vector and $h_{i} j$ is a Gaussian function:

$$
h_{i j}=\exp \left(-\frac{\left\|x_{i}-c_{i j}\right\|}{2 \sigma_{i j}^{2}}\right), \quad i=1,2, j=1,2, \cdots n
$$

where $c_{i j}$ and $\sigma_{i j}$ are respectively the center and base width constants of the $j$-th node of the $i$-th input of the RBFNN, $c_{i j}$ and $\sigma_{i j}$ are numbers greater than zero. 
The rehabilitation robot has parameter uncertainty and external disturbance unpredictability. Therefore, it cannot lead to accurate parameters. Consequently, it is necessary to estimate the optimal parameters of the rehabilitation robot using an adaptive method. $\hat{\xi}_{i}$ is the estimated value of $\xi_{i}^{*}$, while the estimation error of switching gain is $\tilde{\xi}_{i}=\xi_{i}^{*}-\hat{\xi}_{i}$. Note that $\hat{\xi}_{i} \geq 0, i=1,2$. The adaptive gain of sliding mode control can be obtained from the output of the RBFNN:

$$
\hat{\xi}_{i}=\hat{W}_{i}^{T} H_{i}
$$

where $\hat{W}_{i}^{T}$ is the estimated weight vector of the neural network.

The weight adaptive law of the neural network is given by:

$$
\dot{\hat{W}}_{i}=\beta_{i} H_{i}\left|s_{i}\right|, i=1,2
$$

where $\beta_{i}>0$ is the adaptive parameter.

The adaptive RBFNN sliding mode dynamic control law is expressed as:

$$
\tau=\tau_{e q}+\tau_{s w}=B_{0}^{-1}\left(\dot{V}_{c}+\eta e_{c}+\hat{\xi} \operatorname{sgn}(S)\right)
$$

\section{Stability analysis}

The stability proof of the adaptive neural network sliding mode controller. The Lyapunov function is chosen as:

$$
V=\frac{1}{2} s^{T} s+\frac{1}{2} \beta_{1}^{-1} \hat{W}_{1}^{T} \hat{W}_{1}+\frac{1}{2} \beta_{2}^{-1} \hat{W}_{2}^{T} \hat{W}_{2}
$$

The derivation of Equation (22) shows that:

$$
\begin{aligned}
\dot{V} & =s^{T} \dot{s}-\sum_{i=1}^{2} \beta_{i}^{-1} \hat{W}_{i}^{T} \dot{\hat{W}}_{i} \\
& =s^{T}\left(\dot{e}_{c}+\eta e_{c}\right)-\sum_{i=1}^{2} \beta_{i}^{-1} \hat{W}_{i}^{T} \dot{\hat{W}}_{i} \\
& =s^{T}[-\hat{\xi} \operatorname{sgn}(S)-\delta(t)]-\sum_{i=1}^{2} \beta_{i}^{-1} \hat{W}_{i}^{T} \dot{\hat{W}}_{i} \\
& =-\sum_{i=1}^{2}\left\{s_{i}\left[\hat{W}_{i}^{T} H_{i} \operatorname{sgn}\left(S_{i}\right)\right]+s_{i} \delta_{i}(t)+\beta_{i}^{-1} \hat{W}_{i}^{T} \dot{\hat{W}}_{i}\right\} \\
& =-\sum_{i=1}^{2}\left\{\left|s_{i}\right|\left(\hat{W}_{i}^{T} H_{i}-\xi_{i}\right)+\left|s_{i}\right| \xi_{i}+s_{i} \delta_{i}(t)+\beta_{i}^{-1} \hat{W}_{i}^{T} \dot{\hat{W}}_{i}\right\} \\
& =-\sum_{i=1}^{2}\left\{\left|s_{i}\right| \varepsilon_{i}+\left(\left|s_{i}\right| \xi_{i}+s_{i} \delta_{i}(t)\right)\right\} \leq 0
\end{aligned}
$$

where $\varepsilon_{i}>0$, and $\left|s_{i}\right| \xi_{i}+s_{i} \delta_{i}>0$.

$$
\operatorname{dot} V \leq 0
$$

It can be seen in (11) that, if $S=0, e_{c}=-\eta \int_{0}^{t} e_{c}(\tau) d \tau$ is the cue with $e_{c}(\infty) \rightarrow 0$. 


\section{Results}

Experimental design and result analysis

Experimental design of motion control

The following motion control effect of the rehabilitation robot can be evaluated using a quantitative analysis. More precisely, it can be evaluated by controlling the rehabilitation robot to complete the established trajectory and analyzing the position, velocity and angular velocity errors. The "straight line" and "curve" trajectories are the most commonly used. In order to verify the use effect of the robot in the actual environment, two groups of experiments are carried out. The subjects walk along the target track on the ground, and the rehabilitation robot follows the subjects. The robot's walking track is collected, and the pose error between the walking track and the target track is analyzed to judge the robot's following motion control effect. In this paper, two subjects of different gender, age, height and weight are selected in the experiment. The information of the subjects is shown in Table 1, and the experimental site is shown in Figure 11.

Table 1 Motion Control Experiment Volunteer Information

\begin{tabular}{cccccc}
\hline Num & Gender $(\mathrm{f} / \mathrm{m})$ & Age(year) & Height $(\mathrm{cm})$ & Weight $(\mathrm{kg})$ & Remarks \\
\hline 1 & female & 26 & 163 & 58.8 & healthy \\
2 & male & 52 & 171 & 73.6 & patient \\
\hline
\end{tabular}

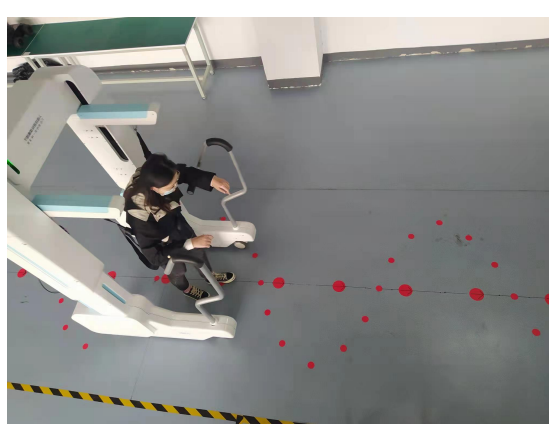

(a)

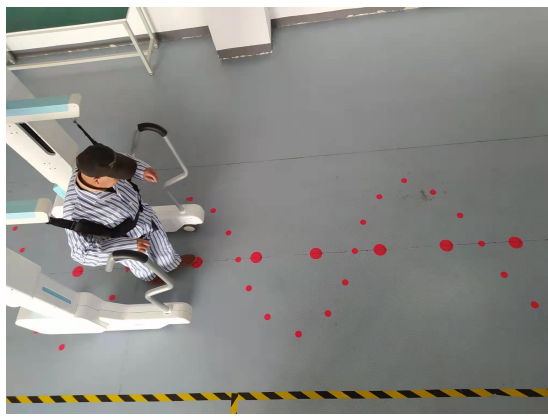

(c)

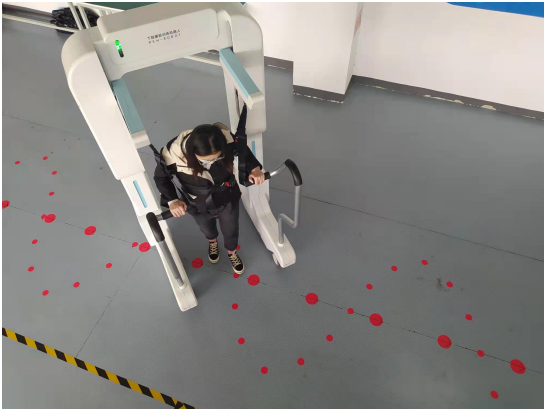

(b)

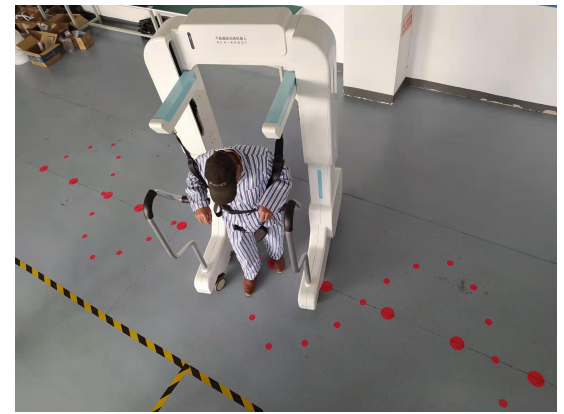

(d)

Figure 11 The picture of following the movement experiment (a) healthy subjects walking in a straight line (b) healthy subjects walking in a curve (c) patients walking in a straight line (d) patients walking in a curve

The robot tracking control performance is evaluated by letting the subjects walk along the given path. The target path of Experiment 1 is a "straight line" track, 
while that of Experiment 2 is a "curve" track. The red dot on the ground is the target trajectory to be tracked. The subjects follow this target trajectory by manipulating the robot.

The mathematical expression of the "straight line" target track is given by:

$$
\left\{\begin{array}{c}
x_{r}=t \\
y_{r}=t \\
\theta_{r}=\pi / 4
\end{array}\right.
$$

The mathematical expression of the curve target track is given by:

$$
\left\{\begin{array}{c}
x_{r}=0.3 * t \\
y_{r}=0.6 * \sin (t) \\
\theta_{r}=0
\end{array}\right.
$$

The subject is required to track the target trajectory by manipulating the robot, and to calculate the position and angle of the rehabilitation robot reference point through the angular velocity value of the driving wheel encoder. The calculation steps are as follows:

Actual running linear speed and angular speed of the rehabilitation robot body:

$$
\left\{\begin{aligned}
v_{r} & =\left(v_{l}+v_{r}\right) / 2 \\
w_{r} & =\left(v_{l}-v_{r}\right) / 2 b
\end{aligned}\right.
$$

The pose of the next moment is calculated according to the pose of the rehabilitation robot at the last moment and equations (25) and (26):

$$
\left\{\begin{array}{c}
\theta_{t}=\theta_{t-1}+w_{r} \\
x_{t}=x_{t-1}+v_{r} \times \cos \theta_{t} \\
y_{t}=y_{t-1}+v_{r} \times \sin \theta_{t}
\end{array}\right.
$$

The following effect of the reference trajectory is evaluated using the integral square error (ISE) normalization method of the pose error evaluation function ERROR. The pose error evaluation function error defines the target pose $\left(x_{r}, y_{r}, \theta_{r}\right)$ and actual pose $\left(x_{t}, y_{t}, \theta_{t}\right)$. The pose error can be expressed as:

$$
E R R O R=\frac{1}{L} \sum_{0}^{t}\left(\left(x_{r}-x_{t}\right)^{2}+\left(y_{r}-y_{t}\right)^{2}+\left(\theta_{r}-\theta_{t}\right)^{2}\right)
$$

where $L$ is the total length of the trajectory.

The experimental results are recorded by the encoder of the driving wheel, and the data are transformed into the position and attitude of the robot reference point through kinematics.

Analysis of experimental results of robot following motion

According to the designed dynamic controller, the following motion experiment is performed on the rehabilitation robot system when the load changes. The control 
mode of the rehabilitation robot is set to automatic, and the auxiliary force provided to the subjects is $24 \mathrm{~kg}$. The healthy subjects complete the motion task within $30 \mathrm{~s}$, while the patients complete the motion task within 60 s. Figures 12 and 13 present the experimental results of two subjects walking along the "straight line" track. It can be seen that the following trend of the experimental track is consistent with the target track, the position error with the target track is small, and the robot can follow two subjects in order to complete the given task. In addition, the pose error evaluation function values of the target trajectory and the experimental trajectory of the two subjects are 5.35 and 6.64 , respectively.

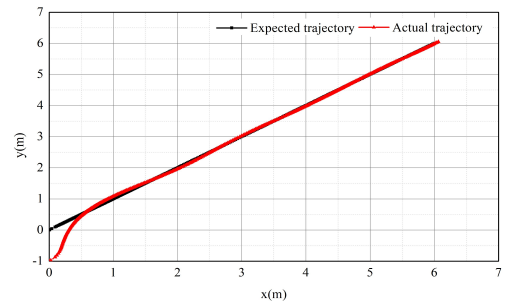

(a)

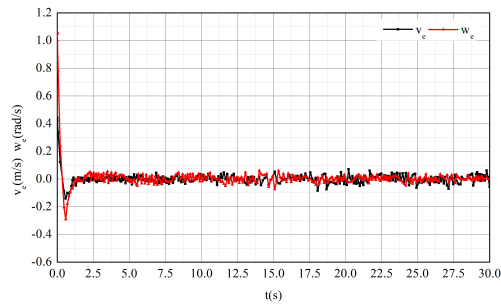

(c)

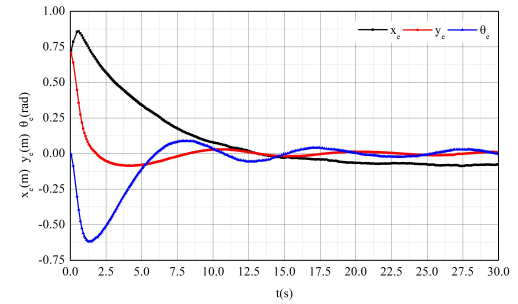

(b)

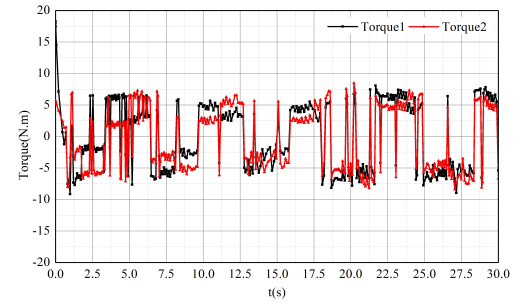

(d)

Figure 12 Follow the experimental curve of linear motion of healthy subjects (a) follow the trajectory (b) pose error (c) speed error (d) control torque

Figures 14 and 15 show the experimental results of two subjects walking along the "curve" track. It can be seen that, when following a more complex track, although the two subjects complete the motion task within the specified time and the trend of the actual walking track is approximately consistent with the target track, obvious pose errors exist. In addition, there is a sudden change in the speed of the mobile platform. However, it does not affect the overall control effect. The pose error evaluation function values of the target trajectory and the experimental trajectory of the two subjects are 36.19 and 72.61 , respectively.

Table 2 shows the comparison results of the calculated values of error evaluation function of subjects under the same "straight line" and "curve" trajectory experimental conditions. The calculation results show that the patients pose error is larger than that of healthy subjects.

Table 2 Motion Control Experiment Volunteer Information

\begin{tabular}{ccc}
\hline Num & 1 & 2 \\
\hline straight line & 5.35 & 6.64 \\
curve & 36.19 & 72.61 \\
\hline
\end{tabular}




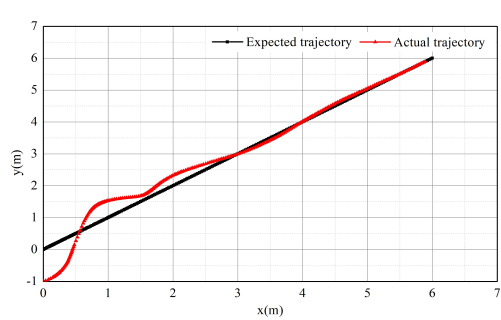

(a)

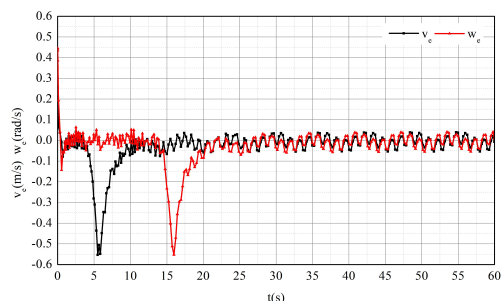

(c)

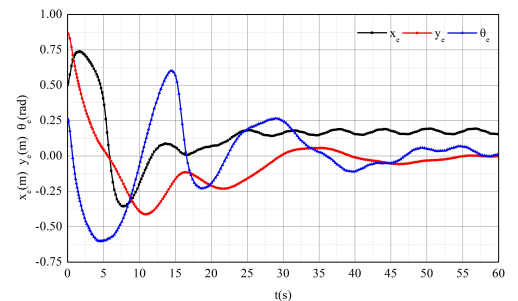

(b)

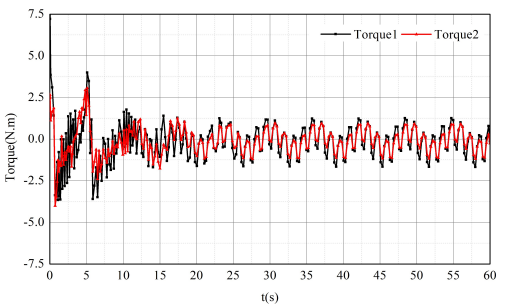

(d)

Figure 13 Follow the experimental curve of linear motion of patients (a) follow the trajectory (b) pose error (c) speed error (d) control torque

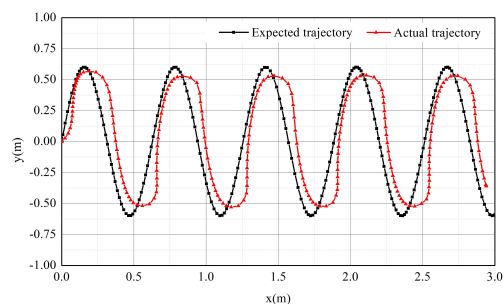

(a)

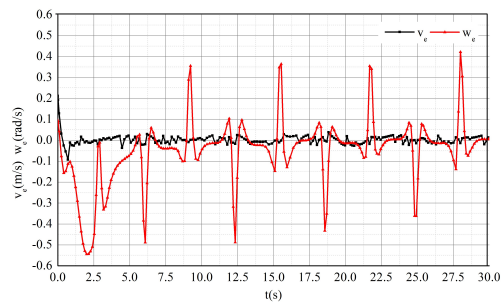

(c)

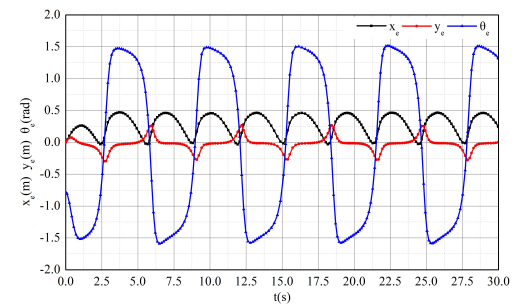

(b)

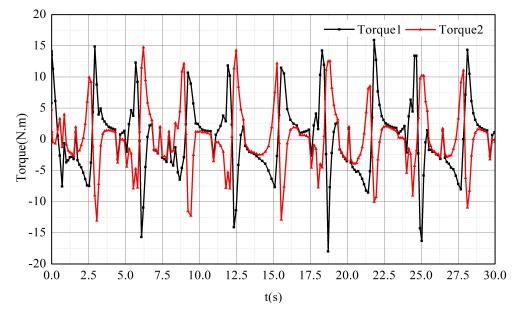

(d)

Figure 14 Follow the experimental curve of curvilinear motion of healthy subjects (a) follow the trajectory (b) pose error (c) speed error (d) control torque

\section{Discussion}

It can be seen from the "straight line" trajectory tracking experimental results of healthy people and patients that, in the "straight line" movement, the body posture of healthy people changes significantly, and the motion characteristics of healthy people are clear and easy to be distinguished. Secondly, the dynamic controller of the robot can adaptively adjust the switching gain of the controller in real time, according to the change characteristics of the healthy person center of gravity 


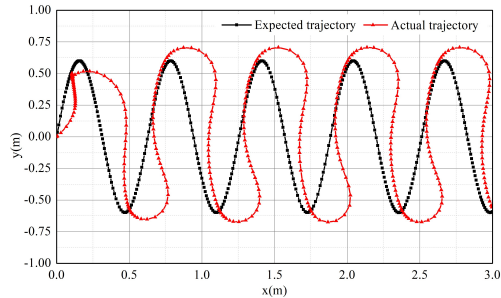

(a)

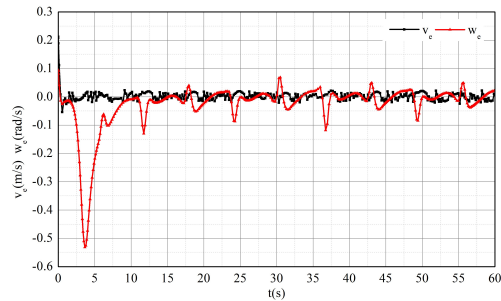

(c)

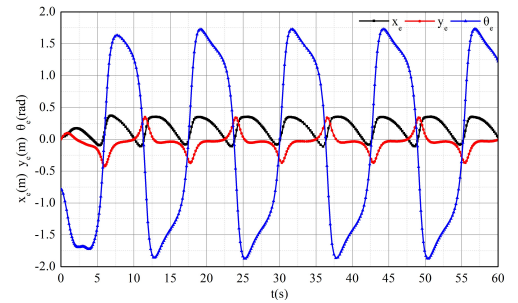

(b)

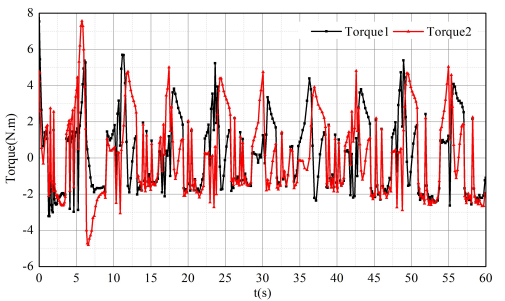

(d)

Figure 15 Follow the experimental curve of curvilinear motion of patients (a) follow the trajectory (b) pose error (c) speed error (d) control torque

when walking. Therefore, the rehabilitation robot can quickly identify the motion posture of healthy people, and then accurately follow the trajectory of healthy people. The pose and velocity errors of the robot are small, and the system can quickly converge to a stable motion state. However, the movement function of the patient's lower limbs is limited, the body coordination is poor, and the movement posture is not clear due to discontinuous walking. Therefore, a certain error exists in the recognition of the patient's movement posture by the robot multi-sensor data acquisition system. The error in the early stage of the robot following the patient's linear movement is clear, and the speed also changes sharply. In addition, when the patient walks, the change range of the center of gravity is greater, and the system disturbance to the rehabilitation robot is also greater. Through the continuous adaptive learning of the control algorithm, the control switching gain of the dynamics is corrected in real time. In the later stage of motion, the pose and speed errors of the robot begin to decrease, and the system gradually tends to a stable following motion state. Although the pose error of the robot following the patient is greater than that of the healthy person, there is no clear difference in the experience of the two subjects in the process of practical application. In addition, the pose error in the straight-line following motion of the robot can be ignored.

In the "curve" movement, the subject needs to continuously adjust the body posture and walk, while the displacement of the displacement sensor changes in real time according to the change of the subject's shoulder posture. According to the "straight line" trajectory tracking experiment, the effectiveness of the proposed ARBFNN sliding mode control algorithm has been proved. However, it can be seen from the "curve" trajectory tracking experimental results of healthy people and patients that there is an obvious deviation in the motion trajectory of the robot following the two subjects along the curve. This indicates that the robot multisensor data acquisition system has a poor recognition effect on the left and right 
turning motion posture of the patients. Since the healthy person and the patient have to respectively complete the task in $30 \mathrm{~s}$ and $60 \mathrm{~s}$, the walking speed of the healthy person is higher than that of the patient, and the motion speed of the rehabilitation robot following the healthy person is also faster, making the robot too fast when following the human body in steering and easy to produce sudden changes in angular velocity. In addition, because the limb reaction speed of the healthy person is better than that of the patient, and the motion characteristics are more obvious when walking along the track. The rotation action is more natural than that of the patient, the rotation angle of the robot following the healthy person is also more regular. Therefore, when the robot follows the subject to turn, the motion response of the healthy person is better than that of the patient. Since the patient's movement is slow and the body's movement during steering is not significant. Although there is no sudden change in angular velocity when the robot turns, there is a significant delay in following the patient's trajectory. Although the error of the rehabilitation robot following the subject's curve motion is greater than the linear motion, the rehabilitation robot can still recognize the motion posture of the healthy person. The position, posture and speed errors of the robot change within a small range. Finally, the system can converge to a stable motion state. The "curve" trajectory tracking experiment also demonstrates that the control algorithm can improve the tracking control effect in human motion tracking control.

In the "straight line" and "curve" trajectory tracking experiments, the robot performed the simple recognition of the subject's motion intention, based on the feedback data of the displacement sensor. The two groups of experiments proved the efficiency of the dynamic model and control algorithm of the mobile platform. However, the follow-up effect of patients was different from that of healthy subjects. The intention recognition algorithm and mobile platform control algorithm should be further improved.

\section{Conclusions}

This paper firstly designs a human-computer interaction data acquisition device based on a multi-sensor system according to the characteristics of the human body's movement behavior. It then analyses the change information of human posture to derive the change pattern and behavioral intention of the human body's center of gravity. In addition, considering the changes of the robot auxiliary force, the influence of the fluctuation of human gravity center on the control system parameters and other external disturbances in the tracking process of the mobile rehabilitation robot, a sliding mode control is introduced. The sliding mode control gain is adjusted using a ARBFNN to eliminate the jitter in the sliding mode control. Finally, the robot following motion experiment is designed. The control experiment, quantitative analysis and evaluation methods are used to test the following motion control effect of the rehabilitation robot on healthy subjects and patients. The experimental results show that the robot can follow different subjects to complete the tasks of straight lines and curves. This proves the correctness of the dynamic modeling method of the mobile platform, and the efficiency of the control algorithm. The algorithm has the characteristics of strong convergence and robustness, which proves that the proposed intelligent control scheme is feasible. However, compared 
with the effect of following healthy subjects, the error of the robot following patient motion is large, in contrast to that of healthy subjects. In future work, because the human-computer interaction data acquisition device of the robot has a better recognition effect on the posture of healthy people than patients, it is crucial to further improve the goodwill map recognition algorithm. We also aim at performing further research on the intention recognition algorithm. Therefore, the robot could accurately identify the motion intention of patients and achieve a better follow-up control effect.

\section{Abbreviations}

ARBFNN:Adaptive radial basis function neural network

\section{Declarations}

Ethics approval and consent to participate

The written informed consent of the participants was obtained for data collection. Participants allowed research team members to access their human motion data to participate in the research project.

Consent for publication

Not applicable.

Availability of data and materials

The datasets used and/or analysed during the current study are available from the corresponding author on reasonable request.

Competing interests

The authors declare that they have no competing interests.

Funding

This work is supported by the National Key R\&D Program of China (GrantNo.2020YFC2008503).

Authors' contributions

Data curation, M.M. and P.Z; Formal analysis, M.M.; Funding acquisition, X.G.; Project administration, X.G. and J.Z; Resources, X.G. and J.Z; Writing-original draft, M.M.; Writing-review \& editing, M.M. and X.G. All authors have read and agreed to the published version of the manuscript.

Acknowledgements

The authors are grateful to Peng Zhao and Hongjuan Che their valuable help in collecting data. The authors would like to express their gratitude to EditSprings (https://www.editsprings.cn/) for the expert linguistic services provided.

Authors' information

School of Mechatronical Engineering, Beijing Institute of Technology, Beijing 100081, China; Department of Neurology, China rehabilitation research center,Beijing 100081, China; School of Electrical Electronic \& Computer Science, Guangxi University of Science and Technology, Liuzhou 545000, China;

\section{Author details}

${ }^{1}$ School of Mechatronical Engineering, Beijing Institute of Technology, Beijing, China. ${ }^{2}$ Department of Neurology, China rehabilitation research center,Beijing, China. ${ }^{3}$ School of Electrical Electronic \& Computer Science, Guangxi University of Science and Technology, Liuzhou, China.

References

1. Gittler, M., Davis, A.M.: Guidelines for adult stroke rehabilitation and recovery. Jama 319(8), 820-821 (2018)

2. Feigin, V.L., Forouzanfar, M.H., Krishnamurthi, R., Mensah, G.A., Connor, M., Bennett, D.A., Moran, A.E., Sacco, R.L., Anderson, L., Truelsen, T., et al.: Global and regional burden of stroke during 1990-2010: findings from the global burden of disease study 2010. The lancet 383(9913), 245-255 (2014)

3. Johnson, C.O., Nguyen, M., Roth, G.A., Nichols, E., Alam, T., Abate, D., Abd-Allah, F., Abdelalim, A., Abraha, H.N., Abu-Rmeileh, N.M., et al.: Global, regional, and national burden of stroke, 1990-2016: a systematic analysis for the global burden of disease study 2016. The Lancet Neurology 18(5), 439-458 (2019)

4. Matarić, M.J., Eriksson, J., Feil-Seifer, D.J., Winstein, C.J.: Socially assistive robotics for post-stroke rehabilitation. Journal of NeuroEngineering and Rehabilitation 4(1), 1-9 (2007)

5. Shi, D., Zhang, W., Zhang, W., Ju, L., Ding, X.: Human-centred adaptive control of lower limb rehabilitation robot based on human-robot interaction dynamic model. Mechanism and Machine Theory 162, 104340 (2021)

6. Bingjing, G., Jianhai, H., Xiangpan, L., Lin, Y.: Human-robot interactive control based on reinforcement learning for gait rehabilitation training robot. International Journal of Advanced Robotic Systems 16(2), $1729881419839584(2019)$ 
7. Cao, J., Xie, S.Q., Das, R., Zhu, G.L.: Control strategies for effective robot assisted gait rehabilitation: the state of art and future prospects. Medical engineering \& physics 36(12), 1555-1566 (2014)

8. Chen, B., Ma, H., Qin, L.-Y., Gao, F., Chan, K.-M., Law, S.-W., Qin, L., Liao, W.-H.: Recent developments and challenges of lower extremity exoskeletons. Journal of Orthopaedic Translation 5, 26-37 (2016)

9. Cespedes, N., Munera, M., Gomez, C., Cifuentes, C.A.: Social human-robot interaction for gait rehabilitation. IEEE Transactions on Neural Systems and Rehabilitation Engineering 28(6), 1299-1307 (2020)

10. Curuk, E., Goyal, N., Aruin, A.S.: The effect of motor and cognitive tasks on gait in people with stroke. Journal of Stroke and Cerebrovascular Diseases 28(11), 104330 (2019)

11. Meng, W., Liu, Q., Zhou, Z., Ai, Q., Sheng, B., Xie, S.S.: Recent development of mechanisms and control strategies for robot-assisted lower limb rehabilitation. Mechatronics 31, 132-145 (2015)

12. Sun, Z., Li, F., Duan, X., Jin, L., Lian, Y., Liu, S., Liu, K.: A novel adaptive iterative learning control approach and human-in-the-loop control pattern for lower limb rehabilitation robot in disturbances environment. Autonomous Robots 45(4), 595-610 (2021)

13. Huang, Y.-C., Hu, H., Chen, C.-H., Chen, Y.-J., Chi, K.-C.: Assistive robot design for lower limbs rehabilitation using fuzzy control. International Journal of Fuzzy Systems, 1-12 (2021)

14. Hu, N., Wang, A., Wu, Y.: Robust adaptive pd-like control of lower limb rehabilitation robot based on human movement data. PeerJ Computer Science 7, 394 (2021)

15. GUO, S., JI, J., MA, G., SONG, T., WANG, J.: Lower limb rehabilitation robot for gait training. Journal of Mechanics in Medicine and Biology 14(06), 1440004 (2014)

16. Zhang, X., Yin, G., Li, H., Dong, R., Hu, H.: An adaptive seamless assist-as-needed control scheme for lower extremity rehabilitation robots. Proceedings of the Institution of Mechanical Engineers, Part I: Journal of Systems and Control Engineering 235(6), 723-734 (2021)

17. Koubaa, Y., Boukattaya, M., Damak, T.: Adaptive sliding-mode control of nonholonomic wheeled mobile robot. In: 2014 15th International Conference on Sciences and Techniques of Automatic Control and Computer Engineering (STA), pp. 336-342 (2014). IEEE

18. Wang, J., Chen, J., Ouyang, S.: Adaptive sliding mode controller with single parameter approximation for four-wheel omnidirectional mobile robots. International Journal of Circuits, Systems and Signal Processing 12, 112-117 (2018)

19. Fan, Q.M., Shu-Hao, L.V.: Adaptive neural network sliding mode control of mobile robots. Control Engineering of China 24, 1409-1414 (2017)

20. Song, L.Y., Xing, F.: Adaptive neural sliding mode trajectory tracking control for mobile robots. Control Engineering of China 25, 1965-1970 (2018)

21. Miao, M., Gao, X., Zhu, W.: A construction method of lower limb rehabilitation robot with remote control system. Applied Sciences 11(2), 867 (2021)

22. Jiang, Y., Larson, J.L.: Ideea activity monitor: validity of activity recognition for lying, reclining, sitting and standing. Frontiers of medicine 7(1), 126-131 (2013)

23. Regnaux, J.-P., Saremi, K., Marehbian, J., Bussel, B., Dobkin, B.H.: An accelerometry-based comparison of 2 robotic assistive devices for treadmill training of gait. Neurorehabilitation and neural repair 22(4), 348-354 (2008)

24. Gardner, M.J., Barker, J.U., Briggs, S.M., Backus, S.I., Helfet, D.L., Lane, J.M., Lorich, D.G.: An evaluation of accuracy and repeatability of a novel gait analysis device. Archives of orthopaedic and trauma surgery 127(3), 223-227 (2007)

25. Saremi, K., Marehbian, J., Yan, X., Regnaux, J.-P., Elashoff, R., Bussel, B., Dobkin, B.H.: Reliability and validity of bilateral thigh and foot accelerometry measures of walking in healthy and hemiparetic subjects. Neurorehabilitation and neural repair 20(2), 297-305 (2006) 